\title{
Functional reach test
}

\section{Functional reach test}

\author{
Dominique Monnin, Simone Gafner, Lara Allet \\ Physiothérapeute, Rue des Marchands 16, 2900 Porrentruy, Suisse \\ dogemo.physio@gmail.com
}

Conflit d'intérêt : Les auteurs déclarent n'avoir aucun conflit d'intérêt en lien avec cet article

\section{- Description}

Le Functional Reach Test (FRT) a été développé par PW Duncan et al. en 1990 [1]. II vise à évaluer les capacité d'équilibration en position debout.

\section{- Utilisation}

\section{Paramètres mesurés}

Le FRT mesure la capacité du sujet à maîtriser son équilibre pendant qu'il se penche le plus possible en avant tout en gardant le bras tendu à l'horizontale. On mesure l'écart de distance en centimètres à l'extrémité distale de la troisième phalange entre la position initiale et la position d'arrivée. Ce paramètre est utilisé comme indicateur de l'équilibre dynamique et du risque de chute.

\section{Déroulement}

Le sujet se tient à proximité d'un mur, sans le toucher, les pieds écartés à la largeur du bassin. Une règle graduée est fixée au mur, à hauteur d'épaule du sujet. Celui-ci tient son bras dominant parallèlement au mur, à $90^{\circ}$ de flexion, coude et poignet étendus, poing fermé. II avance son bras le plus possible, sans décoller les pieds du sol et sans baisser le bras. La distance maximale qu'il peut atteindre correspond à la projection de l'articulation métacarpo- 
phalangienne du 3ème doigt sur le mur [2]. Deux compensations doivent être contrôlées et évitées : une angulation du corps dans le plan sagittal et une rotation du haut du tronc.

Le test peut également être effectué sous enregistrement vidéo [3].

\section{Cotation}

Le sujet effectue trois essais. Duncan et al. [1] proposent de retenir la meilleure performance, mais MacKenzie [4] et Lindmark et al. [5] proposent de retenir la moyenne des trois essais. Le résultat de la variante vidéo est analysé au moyen d'un logiciel dédié [Scena 2016].

\section{Utilisation clinique}

Ce test a été développé pour détecter les limites de la stabilité chez les adultes. L'amplitude de mouvement normale est de $27.2 \mathrm{~cm}$ (IC $95 \%: 25.5$ à 28.9) [6]. Une méta-analyse récente mentionne $26.6 \mathrm{~cm}$, (IC $95 \%: 25.1$ à 28.1) chez des personnes âgées qui vivent à domicile et $15.4 \mathrm{~cm}$ (IC $95 \%$ : 13.5 à 17.4), chez des personnes âgées qui résident en institution [7].

Le risque de chuter dans les six mois est deux fois plus important si la performance se situe entre $15.2 \mathrm{~cm}$ et $25.4 \mathrm{~cm}$, quatre fois plus important si elle se situe à $15.2 \mathrm{~cm}$ ou moins, huit fois plus important chez les personnes qui ne peuvent pas étendre le bras à l'horizontale [8].

\section{Formation}

Pas de formation particulière, mais faire attention aux compensations.

\section{Temps requis}

Environ 5 minutes.

\section{Matériel}

Une marque au sol pour délimiter la position de l'avant des pieds, un mur sur lequel est fixée une toise à hauteur de l'épaule du sujet.

La version vidéo requiert une webcam et un logiciel d'analyse dédié [3].

\section{- Propriétés psychométriques}

\section{Fiabilité}

Lors de la première séance, la fiabilité inter-évaluateur se situe entre ICC : 0.92 (personnes atteintes de déficits cognitifs) [9] et ICC : 0.99 [10]. La fiabilité test-retest (même évaluateur entre deux séances consécutives) varie entre ICC :0.74 chez des patients parkinsoniens [11] et ICC : 0.92 chez des personnes âgées vivant à domicile [1].

\section{Validité}


Le FRT mesure la limite de stabilité du sujet lorsqu'il se penche en avant. Cependant, le test présente une faible corrélation $(r=0.38)$ avec le déplacement du centre de pression; le mouvement du tronc semble plus influencer le résultat que le déplacement du centre de pression [12]. De même, la force des muscles extenseurs et fléchisseurs des hanches semble avoir une corrélation importante avec le résultat du FRT ( $r=0.45$ et $R=0.47$ ) [13].

Utilisés chez des patients hémiparétiques, plateforme de force et FRT semblent mesurer les mêmes capacités de contrôle de l'équilibration [14]. Chez les patients parkinsoniens, le FRT détecte des problèmes de stabilité que l'Unified Parkinson's Disease Rating Scale (UPDRS) ne détecte pas : il est en mesure de prédire des risques d'instabilité posturale [15]. WernickRobinson estime cependant que le FRT ne mesure pas l'équilibration dynamique car des patients atteints de troubles vestibulaires obtiennent des résultats similaires aux personnes en bonne santé [16].

\section{Sensibilité au changement}

La capacité du FRT à détecter un changement semble bonne chez les personnes âgées qui vivent à domicile et qui effectuent divers types d'exercices à sec ou dans l'eau [17] ainsi que chez les patients parkinsoniens [18] ; elle ne l'est pas chez les patients atteints de lésions neurologiques (AVC, TCC) [19], ni chez les patients déficients mentaux [20].

\section{Sensibilité et spécificité}

Le test présente une bonne sensibilité pour identifier les personnes âgées à risque de chutes multiples (sensibilité $63 \%$, spécificité $59 \%$ pour un cut-off à $25 \mathrm{~cm}$ [21]), moins bonne chez les patients parkinsoniens (sensibilité : $30 \%$, spécificité : $92 \%$ pour un cut-off à 25.4 [22].

Aucun effet plancher ou plafond n'est cité dans la littérature. 


\section{Références bibliographiques}

[1] Duncan PW, Weiner DK, Chandler J, Studenski S. Functional reach: a new clinical measure of balance. J Gerontol 1990;45:M192-7.

[2] Weiner DK, Bongiorni DR, Studenski SA, Duncan PW, Kochersberger GG. Does functional reach improve with rehabilitation? Arch Phys Med Rehabil 1993;74:796-800.

[3] Scena S, Steindler R, Ceci M, Zuccaro SM, Carmeli E. Computerized functional reach test to measure balance stability in elderly patients with neurological disorders. J Clin Med Res 2016;8:715-20. doi:10.14740/jocmr2652w.

[4] Mackenzie M. A simplified measure of balance by functional reach. Physiother Res Int 1999;4:233-6. doi:10.1002/pri.168.

[5] Lindmark B, Lagerström C, Naessén T, Larsen H-C, Persson I. Performance in functional balance tests during menopausal hormone replacement: a double-blind placebo-controlled study. Physiother Res Int 1999;4:43-54. doi:10.1002/pri.1999.4.1.43.

[6] Bohannon RW, Wolfson LI, White WB. Functional reach of older adults: normative reference values based on new and published data. Physiotherapy 2017;103:387-91. doi:10.1016/j.physio.2017.03.006.

[7] Rosa MV, Perracini MR, Ricci NA. Usefulness, assessment and normative data of the Functional Reach Test in older adults: A systematic review and meta-analysis. Arch Gerontol Geriatr 2019;81:149-70. doi:10.1016/j.archger.2018.11.015.

[8] VanSwearingen JM, Brach JS. Making geriatric assessment work: selecting useful measures. Phys Ther 2001;81:1233-52.

[9] Rockwood K, Awalt E, Carver D, MacKnight C. Feasibility and measurement properties of the functional reach and the timed up and go tests in the Canadian study of health and aging. J Gerontol A Biol Sci Med Sci 2000;55:M70-73.

[10]Wolf SL, Catlin PA, Gage K, Gurucharri K, Robertson R, Stephen K. Establishing the reliability and validity of measurements of walking time using the Emory Functional Ambulation Profile. Phys Ther 1999;79:1122-33.

[11]Lim LIIK, van Wegen EEH, de Goede CJT, Jones D, Rochester L, Hetherington V, et al. Measuring gait and gait-related activities in Parkinson's patients own home environment: a reliability, responsiveness and feasibility study. Parkinsonism Relat Disord 2005;11:1924. doi:10.1016/j.parkreldis.2004.06.003.

[12]Jonsson E, Henriksson M, Hirschfeld $H$. Does the functional reach test reflect stability limits in elderly people? J Rehabil Med 2003;35:26-30.

[13]Mueller MJ, Salsich GB, Strube MJ. Functional limitations in patients with diabetes and transmetatarsal amputations. Phys Ther 1997;77:937-43.

[14]Cavanaugh JT, Shinberg M, Ray L, Shipp KM, Kuchibhatla M, Schenkman M. Kinematic characterization of standing reach: comparison of younger vs. older subjects. Clin Biomech (Bristol, Avon) 1999;14:271-9.

[15]Jenkins ME, Johnson AM, Holmes JD, Stephenson FF, Spaulding SJ. Predictive validity of the UPDRS postural stability score and the Functional Reach Test, when compared with ecologically valid reaching tasks. Parkinsonism Relat Disord 2010;16:409-11. doi:10.1016/j.parkreldis.2010.04.002.

[16]Wernick-Robinson M, Krebs DE, Giorgetti MM. Functional reach: does it really measure dynamic balance? Arch Phys Med Rehabil 1999;80:262-9.

[17]Dite W, Temple VA. A clinical test of stepping and change of direction to identify multiple falling older adults. Arch Phys Med Rehabil 2002;83:1566-71. 
[18] Stankovic I. The effect of physical therapy on balance of patients with Parkinson's disease. Int J Rehabil Res 2004;27:53-7.

[19]Duncan P, Studenski S, Richards L, Gollub S, Lai SM, Reker D, et al. Randomized clinical trial of therapeutic exercise in subacute stroke. Stroke 2003;34:2173-80. doi:10.1161/01.STR.0000083699.95351.F2.

[20]Carmeli E, Bar-Chad S, Lotan M, Merrick J, Coleman R. Five Clinical Tests to Assess Balance Following Ball Exercises and Treadmill Training in Adult Persons With Intellectual Disability. J Gerontol A Biol Sci Med Sci 2003;58:M767-72. doi:10.1093/gerona/58.8.M767.

[21]Dite W, Temple VA. A clinical test of stepping and change of direction to identify multiple falling older adults. Arch Phys Med Rehabil 2002;83:1566-71.

[22]Behrman AL, Light KE, Flynn SM, Thigpen MT. Is the functional reach test useful for identifying falls risk among individuals with Parkinson's disease? Arch Phys Med Rehabil 2002;83:538-42. 